\title{
Abnormal brain white matter in patients with right trigeminal neuralgia: a diffusion tensor imaging study
}

\author{
Junpeng Liu', Jiajia Zhu'², Fei Yuan ${ }^{3}$, Xuejun Zhang ${ }^{1^{*}}$ (D) and Quan Zhang ${ }^{3^{*}}$
}

\begin{abstract}
Background: Idiopathic or classical trigeminal neuralgia (TN) is a chronic painful condition characterized by intermittent pain attacks. Enough evidence demonstrates classical TN is related to neurovascular compression (NVC) at the trigeminal root entry zone (REZ), but white matter change secondary to TN are not totally known.

Methods: Visual Analogue Scale (VAS) and diffusion tensor imaging were performed on 29 patients with right TN and 35 healthy individuals. Voxel-wise analyses were performed with TBSS using multiple diffusion metrics, including fractional anisotropy (FA), mean diffusivity (MD), axial diffusivity (AD) and radial diffusivity (RD). Group differences in these parameters were compared between right TN patients and controls using TBSS and correlations between the white matter change and disease duration and VAS in right TN patients were assessed. Multiple comparison correction were applied to test significant correlations.

Results: The right TN patients showed significantly lower FA and higher RD in most left white matter $(P<0.05$, FWE corrected). Moreover, negative correlations were observed between disease duration and the FA values of left corona radiata, genu of corpus callosum, left external capsule and left cerebral peduncle, and between VAS and the FA values of left corona radiata, left external capsule and left cerebral peduncle $(P<0.05)$. Positive correlations were observed for disease duration and the RD values of left corona radiata, right external capsule, left fornix cerebri and left cerebral peduncle, and for VAS and the RD values of left corona radiata and left external capsule $(P<0.05)$. However, once Bonferroni corrections were applied, these correlations were not statistically significant.

Conclusion: These findings suggest that TN selectively impairs widespread white matter, especially contralateral hemisphere, which may be the hallmark of disease severity in TN patients.
\end{abstract}

Keywords: Trigeminal neuralgia, Neurovascular compression, Magnetic resonance imaging, Tract-based spatial statistics, White matter

\section{Background}

Trigeminal neuralgia (TN) is the most common form of facial neuropathic pain with an annual incidence of 4 to 5 new patients per 100,000 [1]. It is characterized by recurrent episodes of unilateral brief electric shock-like pains localized to the sensory supply areas of trigeminal

\footnotetext{
* Correspondence: zhangxj@tmu.edu.cn; tj_zhangquan1981@163.com ${ }^{1}$ School of Medical Imaging, Tianjin Medical University, No. 1, Guangdong Road, Hexi District, Tianjin 300203, China

${ }^{3}$ Department of Radiology, Pingjin Hospital, Logistics University of Chinese People's Armed Police Forces, No. 220, Chenglin Road, Hedong District, Tianjin 300162, China

Full list of author information is available at the end of the article
}

nerve and has been considered as one of the most serious pains that can experience [2-4]. Idiopathic or classical TN is mainly caused by neurovascular compression of trigeminal nerve at its root entry zone (REZ) and microvascular decompression (MVD) surgery is most effective method for relieving neuralgic pain [3-7]. However, peripheral nerve injury caused by neurovascular compression does not fully explain the persistence of long-term recurrent pain in TN patients [8-11].

Neurovascular compression may result in focal demyelination of the trigeminal nerve at the REZ, which consequently generates ectopic discharges and pathological 
cross-activation between afferent nerve fibers [12]. And pain ensues. What's more, this process may lead to central white matter changes and/or higher brain structures and sensitization of neurons [13-15]. Previous studies have mostly concentrated on abnormalities of trigeminal nerve [16], but the nature of assumed nerve abnormalities is not known. Diffusion tensor imaging (DTI) based on magnetic resonance imaging (MRI) has been considered as a useful and effective examination of the trigeminal nerve system in great detail $[8,12,17]$.

Previous studies have demonstrated white matter abnormalities due to chronic pain and peripheral nerve damage in the TN patients $[8,16,18]$. The results of these studies are similar. These studies demonstrate significantly decreased FA and increased AD, RD and MD. However, the mechanism of TN affecting brain white matter remains unclear.

In order to further understand the relationship between TN and brain white matter plasticity, we examined white matter microstructural change and correlation of between white matter abnormality and disease duration and pain intensity in patients with classical TN.

\section{Methods}

\section{Participants}

Twenty-nine patients (age range 35-77 years; 20 females and 9 males) with right-sided TN and 35 healthy control subjects (age range 41-74 years; 27 females and 8 males) were selected for this study. Patients were enrolled from Department of Functional Neurosurgery of Pingjin Hospital of Logistics University of Chinese People's Armed Police Forces (Additional file 1: Table S1) and control subjects by newspaper advertisement. The patients belonged to a consecutive series of patients who had undergone evaluation for MVD surgery between 2014 and 2016. All these patients had a long duration (more than 1 year) complaint of classical TN according to the International Classification of Headache Disorders criteria (third edition) [19] and had high-resolution imaging to exclude secondary causes of TN. Visual analogue scale (VAS) [20] was used to assess pain intensity in the TN patients. All patients were measured during a painful attack and on medications. Exclusion criteria [18] for both the patients and controls were as follows: (1) other headache disorders; (2) chronic pain elsewhere; (3) previous TN operations; (4) untreated hypertension or diabetes mellitus; (5) left-handed; (6) alcohol or illicit drug abuse, or current intake of psychoactive medications; and (7) MRI contraindications, such as claustrophobia and metallic implants or devices in the body. This study was approved by our institutional review board, and written informed consent was obtained from all patients and control subjects.

\section{MRI data acquisition}

DTI data were obtained using a 3.0-T MR scanner (Verio system; Siemens, Erlangen, Germany) with a 12-channel head coil. Comfortable and tight foam padding was used to limit head movement. Diffusion weighted images were obtained using a single-shot echo planar imaging (EPI) sequence. The scanning location was in alignment with the anterior-posterior commissural plane. The integral parallel acquisition technique (iPAT) was used and the acceleration factor was 2, which can decrease image distortion from susceptibility artifacts. Diffusion sensitizing gradients were applied along 64 non-collinear directions $\left(b=1000 \mathrm{~s} / \mathrm{mm}^{2}\right)$ together with an acquisition without diffusion weighting $(b=0 \mathrm{~s} /$ $\mathrm{mm}^{2}$ ). The imaging parameters were applied as follows: 48 continuous axial slices, slice thickness of $3 \mathrm{~mm}$ and no gap, field of view $(F O V)=256 \mathrm{~mm} \times 256 \mathrm{~mm}$, repetition time/echo time $(\mathrm{TR} / \mathrm{TE})=8000 / 95 \mathrm{~ms}$, and matrix size $=128 \times 128$. The reconstruction matrix was $256 \times$ 256 with a voxel dimension of $1 \mathrm{~mm} \times 1 \mathrm{~mm} \times 3 \mathrm{~mm}$.

\section{Data preprocessing}

All diffusion weighted images were carefully checked by three radiologists to exclude apparent artifacts resulted from instrument malfunction and subject motion. DTI data was preprocessed using FMRIB's diffusion toolbox (FDT, http://www.fmrib.ox.ac.uk/fsl, FSL 4.0) [21]. First, the eddy current distortions and motion artifacts were corrected by using the affine alignment of each diffusion-weighted image to the image of $b=0 \mathrm{~s} / \mathrm{mm}^{2}$ in the DTI dataset. Then, non-brain tissue from the average b0 image was removed using the FMRIB's Brain Extraction Toolbox, BET. The brain mask was applied to the rest of the diffusion-weighted images. Finally, the diffusion tensor was estimated for each voxel using the DTIFIT function via linear regression to derive FA, MD, $\mathrm{AD}$ and RD maps.

\section{Tract-based spatial statistics (TBSS)}

The following steps were adopted to perform voxel-wise analysis of whole brain white matter measures using the TBSS package (http://www.fmrib.ox.ac .uk/fsl/tbss/index.html) [22]. All subjects' FA images were aligned to a template of the averaged FA images (FMRIB-58) in Montreal Neurological Institute (MNI) space using a nonlinear registration algorithm implemented in FNIRT (FMRIB's nonlinear registration Tool) [23]. After transformation into the MNI space, a mean FA image was generated and thinned to create a mean FA skeleton of white matter tracts. Each subject's aligned FA images were then projected onto the mean FA skeleton according to filling the mean FA skeleton by FA values, resulting in an alignment-invariant representation of the central trajectory of white matter pathways for all 
subjects. These FA values were obtained by searching perpendicular to the local skeleton structure for maximum value, which was from the nearest relevant tract center. During the former registration step, this second local coregistration step can alleviate the malalignment of diffusion-weighted images. Next, this process was repeated for each subject's $M D, A D$ and $\mathrm{RD}$ map using the individual registration and projection vectors obtained in the FA nonlinear registration and skeletonization.

\section{Statistical analyses}

Voxel-wise differences in FA, MD, AD and RD values of white matter between TN patients and healthy controls were tested using a permutation-based inference tool by nonparametric statistic ("randomize", part of FSL) and two-sample t-tests. The mean FA skeleton was applied as a mask (thresholded at a mean FA value of 0.2 to include only major fiber bundles and exclude peripheral tracts with significant intersubject variability), and the number of permutations was set to 5000 to allow robust statistical inference. Age and gender were entered into the analysis as confound regressors. The significance threshold for intergroup differences was $P<0.05$ after correcting for family wise error (FWE) applying the threshold-free cluster enhancement (TFCE) option by permutation-testing tool in FSL. The white matter tracts were identified using the Johns Hopkins University ICBM-DTI-81 White-Matter Labels provided in the FSL toolbox. In addition, significant white matter clusters were described by their coordinates in MNI convention and by their cluster size.

To study the relationship between clinical variables (disease duration and VAS) and each of the DTI measures, region-of-interest- (ROI-) based correlation analyses was performed by using a partial correlation $(P$ $<0.05)$. Regional ROI masks were created for brain sites using clusters determined by voxel-by-voxel intergroup analysis procedures mentioned above. After the extraction of each ROI, the mean FA, MD, AD or RD value of the ROI were calculated. Finally, the correlations were calculated between the DTI measures of each ROI and disease duration and VAS with age and gender as covariates of no interest. Because so many correlations were run, the Bonferroni correction was applied to correct for multiple correlation comparisons.

The demographic and clinical data were compared between the two groups using independent-sample $t$-test for age and Chi-square test for sex distribution, which was conducted with Statistical Package for the Social Sciences version 22.0 (SPSS, Chicago, Ill, USA). Differences were considered significant when $P$ was less than 0.05 .

\section{Results}

\section{Demographic and clinical data}

In our study, 35 patients with right-sided TN were enrolled. Due to the abnormal data quality and no surgery, 6 patients were excluded in this study. Therefore, 29 patients (age range 35-77 years; 20 females and 9 males) and 35 healthy control subjects (age range 41-74 years; 27 females and 8 males) were selected for this study. Demographic and clinical characteristics of each group are summarized in Additional file 1: Table S2. The self-reported duration in TN patients was $10.2 \pm 9.6$ years (range: $1-30$ years) and the VAS was $5.9 \pm 3.1$ (range: $2-$ $10)$. There were no significant differences $(P>0.05)$ between TN patients and healthy controls in age and gender.

\section{Comparison of DTI metrics between TN and controls}

Compared with the control group, the TN group showed significantly lower FA in the bilateral superior corona radiata, bilateral anterior corona radiata, body of corpus callosum, splenium of corpus callosum, genu of corpus callosum, left cingulum, left superior fronto-occipital fasciculus, bilateral anterior limb of internal capsule, left posterior limb of internal capsule, left external capsule, left fornix cerebri, internal sagittal stratum and left cerebral peduncle $(P<0.05$, FWE corrected) (Fig. 1; Additional file 1: Table S3). Moreover, the TN group demonstrated higher RD in the bilateral superior corona radiata, bilateral anterior corona radiata, body of corpus callosum, splenium of corpus callosum, left cingulum, left superior fronto-occipital fasciculus, bilateral anterior limb of internal capsule, bilateral posterior limb of internal capsule, bilateral external capsule, left retrolenticular portion, left fornix cerebri, pontine crossing tract, corticospinal tract and left cerebral peduncle $(P<0.05$, FWE corrected) (Additional file 1: Figure S1, Table S3). However, no significant difference was found in MD and $\mathrm{AD}$ between the TN group and control group.

\section{Correlations between clinical variables and altered DTI metrics}

In the TN patients, negative correlations were observed between disease duration and the FA values of left anterior corona radiata $(r=0.211, P=0.012)$, genu of corpus callosum $(r=0.166, P=0.028)$, left external capsule $(r=$ $0.190, P=0.018)$, left cerebral peduncle $(r=0.192, P=$ $0.017)$, and between VAS and the FA values of left anterior corona radiata $(r=0.221, P=0.010)$, left external capsule $(r=0.218, P=0.011)$, left cerebral peduncle $(r=$ $0.168, P=0.027$ ) (Fig. 2). Positive correlations were observed for disease duration and the RD values of left anterior corona radiata $(r=0.190, P=0.018)$, right external capsule $(r=0.170, P=0.026)$, left fornix cerebri $(r=$ $0.168, P=0.027)$, left cerebral peduncle $(r=0.156, P=$ 


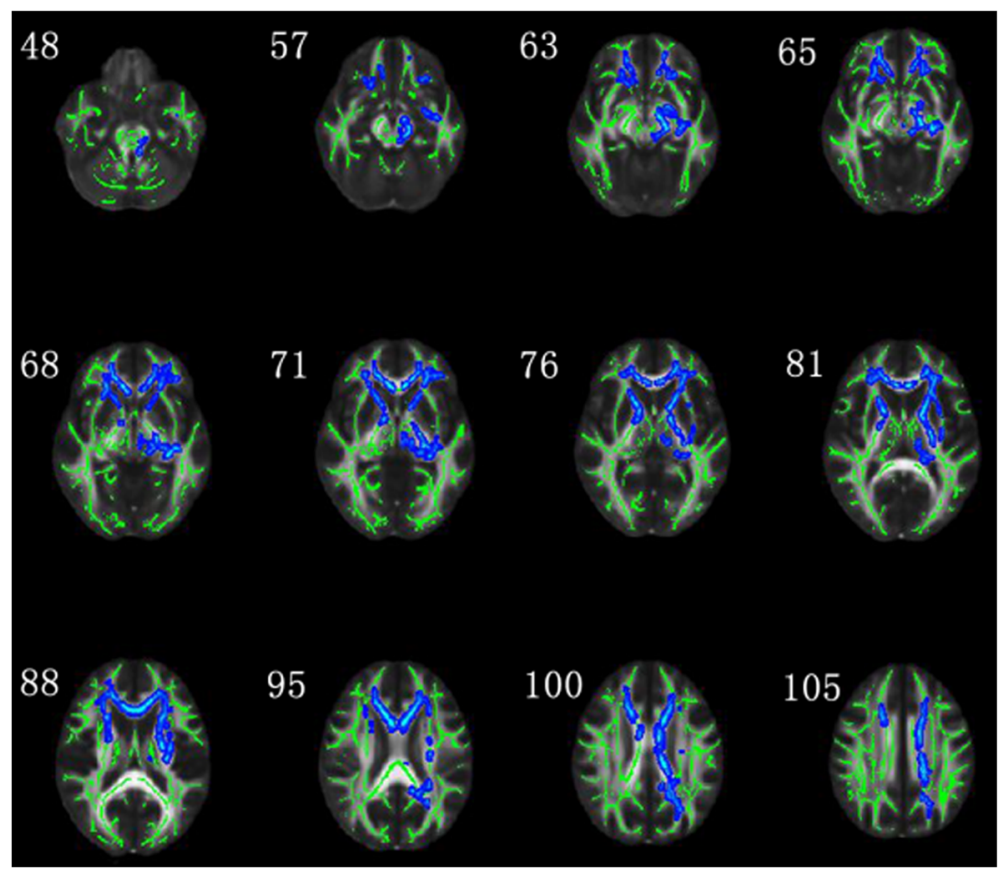

Fig. 1 TBSS shows white matter regions with significant differences in FA between TN patients and healthy subjects $(P<0.05$, FWE corrected). Green represents mean FA skeleton of all participants; blue represents reduction in right TN patients. Coordinates are in millimeters along $z$ axe

0.034), and for VAS and the RD values of left anterior corona radiata $(r=0.174, P=0.025)$, left external capsule $(r=0.191, P=0.018)$ (Additional file 1: Figure S2). However, once Bonferroni corrections were applied, these correlations were not statistically significant.

\section{Discussion}

\section{Methological consideration}

Compared with voxel-based analysis (VBA), the TBSS method is applied more and more popularly to reveal microstructural alterations of white matter fibers between groups [24-27]. VBA has two severe limitations about different subjects' alignment of FA images and the self-willed choice of smoothing kernels without any principle or standard [28]. TBSS solve these issues using carefully tuned non-linear registration, and then projecting onto the "mean FA skeleton"(an alignment-invariant tract representation). Moreover, TBSS doesn't need a smoothing process [22]. Therefore, TBSS can avoid the two limitations and provides us with more diffusion metrics [29]. In many disease studies, TBSS has a wide application to research microstructural white matter alterations, such as adiposity, parkinsonism, Alzheimer's disease (AD), genetic disease, type2 diabetes mellitus and so on [30-35]. In this study, we have been showed well results of the diffusion metrics (FA, MD, AD and RD) in TBSS methods between groups.

\section{White matter impairment in TN patients}

The primary diffusion metrics (FA and MD) reflect overall white matter health, organization and maturation [36]. In addition, AD reflects axon integrity and RD reflects myelin sheath integrity [31]. Both AD and RD are of great significance in understanding the underlying physiological mechanism [26]. Decreased FA values maybe based on predominantly increase of RD or both $\mathrm{RD}$ and $\mathrm{AD}$ [30]. The study of DeSouza et al. showed the right-sided $\mathrm{TN}$ patients had significantly decreased FA and increased $\mathrm{RD}, \mathrm{MD}$, and $\mathrm{AD}$ in the brain white matter including the corpus callosum, posterior corona radiata, cingulum, and superior longitudinal fasciculus. Moreover, MD and RD changes of brain white matter in TN patients maybe have relation to central nervous system plasticity, neuroinflammation and edema [18]. In our study, we found the similar results that reduced FA and elevated RD in the corona radiata (mainly concentrating on bilateral superior corona radiate and anterior corona radiata), corpus callosum and left cingulum. Additionally, we found reduced FA and elevated RD in the fronto-occipital fasciculus, internal capsule, external capsule, fornix cerebri and cerebral peduncle in the TN patients. Moreover, altered FA and RD was mainly located in white matter of left hemisphere. However, the differences of MD and AD were not statistically significant. As we all know, TN is involved in trigeminal nerve functional disorders, but other theories of central nervous system pathology is not clear [18, 37]. Due to 


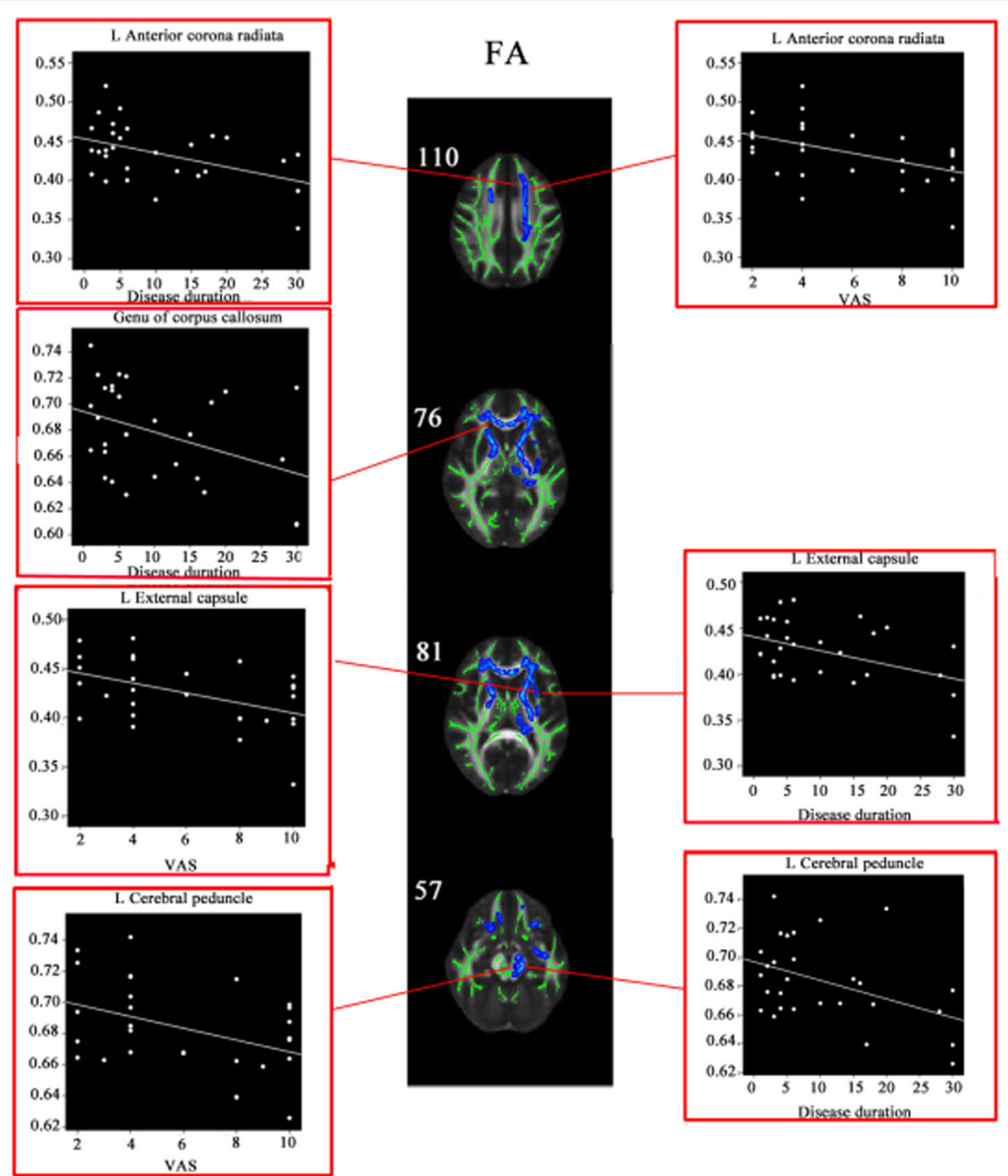

Fig. 2 Correlation between the decreased FA and disease duration and VAS. Coordinates are in millimeters along $z$ axe

peripheral trigeminal nerve injury, central nervous system plasticity will most probably occur $[18,38,39]$, including fiber organization changes, astrocyte morphology and angiogenesis [18, 40-42]. In our study, compared with healthy controls, TN patients showed decreased FA. The decreased FA may correspond to less fiber organization, such as more axonal sprouting/branching, larger axons, or more crossing fibers $[18,42]$. Besides, neuropathic pain is usually related to chronic painful influence on central nervous system. This leads to central sensitization, a process involving demyelination and neuroinflammation processes [18]. The decreased FA is presumably caused by a significant increase of RD, inferring that demyelination and neuroinflammation processes may lead to the impairment of white matter integrity in the TN patients. The RD abnormalities maybe result from these mechanisms in the white matter of TN patients [8].
Many studies have reported cortical and subcortical gray matter impairment in the cognitive-affective, sensory, modulation of pain, attention and motor regions of TN patients [43-45]. In anatomy and/or function respect, these brain areas are connected [13, 46-48]. In our study, we reported decreased FA and increased RD in the corona radiata, corpus callosum, cingulum, fronto-occipital fasciculus, internal capsule, external capsule, fornix cerebri and cerebral peduncle in the TN patients. These fiber connection of brain regions is related to rapid transmission of pain, attention and motor function [49], and maybe lead to the unique sensory symptoms of TN. Our finding also revealed that altered FA and RD was mainly located in white matter of left hemisphere, suggesting contralateral white matter lesions of $\mathrm{TN}$ patients. 
Correlation analyses found negative correlations between the disease duration and the FA values of left anterior corona radiata, genu of corpus callosum, left external capsule, left cerebral peduncle, indicating the white matter impairment is more and more severe as the disease progressed. With impairing progression of left anterior corona radiata, left external capsule and left cerebral peduncle, painful sensation is more serious. What is more, we infer these regions are probably related to transmission of pain [50]. The RD and the disease duration also reveal positive correlations in the regions of left anterior corona radiata, right external capsule, left fornix cerebri, left cerebral peduncle, demonstrating the white matter demyelination and neuroinflammation of these regions is aggravated in the disease progression. And as demyelination and neuroinflammation of left anterior corona radiate and left external capsule progresses, painful sensation is also more serious. Regrettably, the results of these correlation analyses had not been able to withstand multiple comparison correction.

\section{Conclusion}

In our study, we revealed directly differences between the healthy control and right TN to demonstrate how brain white matter is changed using TBSS methods, suggesting that white matter impairment is the significant hallmark in the right TN. Moreover, the correlation analyses between FA/RD and the disease duration and VAS indicate white matter impairment is more and more severe in the disease progression. And the pain is also more serious with some regions of white matter impairment. The white matter impairment is mostly based on fiber organization, demyelination and neuroinflammation. So we can deeply understand the mechanism of white matter change of $\mathrm{TN}$ patients.

\section{Limitations}

Several limitations should be considered when interpreting our results. First, the sample size of the present study was not much, which might cause correlation analyses to fail to withstand multiple comparison correction. Second, non-isotropic voxels were used for DTI data acquisition in this study. In terms of the tensor evaluation, isotropic voxels are more accurate than non-isotropic voxels. Finally, all patients in our study were on medications for $\mathrm{TN}$ pain and the anticonvulsant carbamazepine is the most common. The influences of antiepileptics on brain structure are not clear. Future studies are needed to collect more sample sizes, adopt more optimized DTI parameters and avoid the effects of drugs.

\section{Additional file}

Additional file 1: Table S1. Characteristics and findings in 29 right TN patients who underwent MVD. Table S2. Demographic and clinical data for TN patients and healthy controls. Table S3. Comparison of DTI metrics between TN and controls. Figure S1. TBSS shows white matter regions with significant differences in $\mathrm{RD}$ between TN patients and healthy subjects ( $P<0.05$, FWE corrected). Green represents mean FA skeleton of all participants; red denotes increase in right TN patients. Coordinates are in millimeters along z axe. Figure S2. Correlation between the increased RD and disease duration and VAS. Coordinates are in millimeters along $z$ axe. (DOC $1102 \mathrm{~kb}$ )

\begin{abstract}
Abbreviations
AD: Axial diffusivity; DTI: Diffusion tensor imaging; EPI: Echo planar imaging; FA: Fractional anisotropy; FDT: FMRIB's diffusion toolbox; FOV: Field of view; iPAT: Integral parallel acquisition technique; MD: Mean diffusivity; MNI: Montreal Neurological Institute; MRI: Magnetic resonance imaging MVD: Microvascular decompression; NVC: Neurovascular compression; RD: Radial diffusivity; REZ: Root entry zone; ROI: Region-of-interest; SPSS: Statistical package for the social sciences; TFCE: Threshold-free cluster enhancement; TN: Trigeminal neuralgia; VAS: Visual analogue scale/score; VBA: Voxel-based analysis
\end{abstract}

\section{Acknowledgments}

We thank Dr. Hongtao Sun (Department of Functional Neurosurgery of Pingjin Hospital, Logistics University of Chinese People's Armed Police Forces, Tianjin, China) for his help in the recruitment of patients; and Dr. Kewen Al (Department of Radiology of Pingjin Hospital, Logistics University of Chinese People's Armed Police Forces, Tianjin, China) for his help in the recruitment of healthy volunteers.

\section{Funding}

This study was supported by the Natural Science Foundation of China (No. 81401401), the Natural Science Foundation of Tianjin (Nos. 16JCQNJC10900 and 16JCZDJC36000) and the Science Fund of Tianjin Medical University (No. 2014KYQ11).

Availability of data and materials

All the data supporting our findings is contained within the manuscript.

\section{Declarations}

No financial support has been directly or indirectly received for the ideation, preparation and in writing this paper.

\section{Authors' contributions}

QZ was the project leader and contributed to project design and development of the methodology. FY enrolled and evaluated the patients. JL and JZ collected and analyzed the imaging data. $J$ drafted the manuscript. QZ and $X Z$ revised the manuscript critically. All authors read and approved the final manuscript.

\section{Ethics approval and consent to participate}

The Institutional Review Board is the Pingjin Hospital, Logistics University of Chinese People's Armed Police Forces, Tianjin, China. This institution approved the research protocol, and each participant provided written informed consent.

\section{Consent for publication}

Not applicable.

\section{Competing interests}

The authors declare that they have no competing interests.

\section{Publisher's Note}

Springer Nature remains neutral with regard to jurisdictional claims in published maps and institutional affiliations. 


\section{Author details}

'School of Medical Imaging, Tianjin Medical University, No. 1, Guangdong Road, Hexi District, Tianjin 300203, China. ${ }^{2}$ Department of Radiology, The First Affiliated Hospital of Anhui Medical University, Hefei, China. ${ }^{3}$ Department of Radiology, Pingjin Hospital, Logistics University of Chinese People's Armed Police Forces, No. 220, Chenglin Road, Hedong District, Tianjin 300162, China.

Received: 5 March 2018 Accepted: 13 June 2018

Published online: 22 June 2018

\section{References}

1. Bescos A, Pascual V, Escosa-Bage MMalaga X (2015) Treatment of trigeminal neuralgia: an update and future prospects of percutaneous techniques. Rev Neurol 61:114-124

2. Zakrzewska JM (2002) Diagnosis and differential diagnosis of trigeminal neuralgia. Clin J Pain 18:14-21.

3. Wankel I, Dietrich U, Oppel FPuchner MJA (2005) Endovascular treatment of trigeminal neuralgia caused by arteriovenous malformation: is surgery really necessary? Zbl Neurochir 66:213-216.

4. Hayashi M (2009) Trigeminal neuralgia. Prog Neurol Surg 22:182-190.

5. Yoshino N, Akimoto H, Yamada I, Nagaoka T, Tetsumura A, Kurabayashi T et al (2003) Trigeminal neuralgia: evaluation of neuralgic manifestation and site of neurovascular compression with 3D CISS MR imaging and MR angiography. Radiology 228:539-545.

6. Tien RD, Wilkins RH (1993) MRA delineation of the vertebral-basilar system in patients with hemifacial spasm and trigeminal neuralgia. AJNR. Am J Neuroradiol 14:34-36.

7. Sens MA, Higer HP (1991) MRI of trigeminal neuralgia: initial clinical results in patients with vascular compression of the trigeminal nerve. Neurosurg Rev 14:69-73.

8. Liu Y, Li J, Butzkueven H, Duan Y, Zhang M, Shu N et al (2013) Microstructural abnormalities in the trigeminal nerves of patients with trigeminal neuralgia revealed by multiple diffusion metrics. Eur J Radiol 82 783-786.

9. Leclerca D, Thiebaut JB, Heran F (2013) Trigeminal neuralgia. Diagn Interv Imaging 94:993-1001.

10. Fahlstrom A, Laurell K, Ericson H (2014) Trigeminal neuralgia. Lakartidningen 111:2295-2298.

11. Cruccu G (2017) Trigeminal Neuralgia. Continuum (Minneapolis, Minn) 23: 396-420.

12. Neetu S, Sunil K, Ashish A, Jayantee K, Usha Kant M (2016) Microstructural abnormalities of the trigeminal nerve by diffusion-tensor imaging in trigeminal neuralgia without neurovascular compression. Neuroradiol J 29: 13-18.

13. Scrivani $S$, Moulton E, Pendse $G$, Morris $S$, Aiello-Larnmens $M$, Beccera $L$ et al (2008) Functional magnetic resonance imaging (IMRI) of evoked and spontaneous painful tics in trigeminal neuralgia (tic doloureux). Headache 48:S11-S11.

14. Naegel S, Holle D, Gaul C, Gizewski ER, Diener HC, Katsarava Z et al (2009) Event-related fMRI activation in spontaneous trigeminal neuralgia attacks. Cephalalgia 29:115-116.

15. Blatow M, Nennig E, Sarpaczki E, Reinhardt J, Schlieter M, Herweh C et al (2009) Altered somatosensory processing in trigeminal neuralgia. Hum Brain Mapp 30:3495-3508.

16. Wang Y, Li D, Bao F, Guo C, Ma S, Zhang M (2016) Microstructural abnormalities of the trigeminal nerve correlate with pain severity and concomitant emotional dysfunctions in idiopathic trigeminal neuralgia: a randomized, prospective, double-blind study. Magn Reson Imaging 34:609-616.

17. Lacerda Leal PR, Roch JA, Hermier M, Nobre Souza MA, Cristino-Filho G, Sindou M (2011) Structural abnormalities of the trigeminal root revealed by diffusion tensor imaging in patients with trigeminal neuralgia caused by neurovascular compression: a prospective, double-blind, controlled study. Pain 152:2357-2364

18. DeSouza DD, Hodaie M, Davis KD (2014) Abnormal trigeminal nerve microstructure and brain white matter in idiopathic trigeminal neuralgia. Pain 155:37-44.

19. Cho SJ, Kim BK, Kim BS, Kim JM, Kim SK, Moon HS et al (2016) Vestibular migraine in multicenter neurology clinics according to the appendix criteria in the third beta edition of the international classification of headache disorders. Cephalalgia 36:454-462.
20. Li X, Lin CN, Liu CC, Ke SJ, Wan Q, Luo HJ et al (2017) Comparison of the effectiveness of resistance training in women with chronic computerrelated neck pain: a randomized controlled study. Int Arch Occ Env Hea 90: 673-683.

21. Smith SM, Jenkinson M, Woolrich MW, Beckmann CF, Behrens TEJ, Johansen-Berg $\mathrm{H}$ et al (2004) Advances in functional and structural MR image analysis and implementation as FSL. Neuroimage 23:S208-SS19.

22. Smith SM, Jenkinson M, Johansen-Berg H, Rueckert D, Nichols TE, Mackay CE et al (2006) Tract-based spatial statistics: Voxelwise analysis of multisubject diffusion data. Neuroimage 31:1487-1505.

23. Rueckert D, Sonoda LI, Hayes C, Hill DLG, Leach MO, Hawkes DJ (1999) Nonrigid registration using free-form deformations: application to breast MR images. leee T Med Imaging 18:712-721.

24. Sage CA, Van Hecke W, Peeters R, Sijbers J, Robberecht W, Parizel P et al (2009) Quantitative diffusion tensor imaging in amyotrophic lateral sclerosis: revisited. Hum Brain Mapp 30:3657-3675.

25. Huang R, Lu M, Song Z, Wang J (2015) Long-term intensive training induced brain structural changes in world class gymnasts. Brain Struct Funct 220:625-644.

26. Hasan KM, Walimuni IS, Abid H, Hahn KR (2011) A review of diffusion tensor magnetic resonance imaging computational methods and software tools. Comput Biol Med 41:1062-1072.

27. Duan F, Zhao T, He Y, Shu N (2015) Test-retest reliability of diffusion measures in cerebral white matter: a multiband diffusion MRI study. J Magn Reson Imaging 42:1106-1116.

28. Wang D, Qin W, Liu Y, Zhang Y, Jiang T, Yu C (2013) Altered white matter integrity in the congenital and late blind people. Neural Plasticity 2013: 128236

29. Bach M, Laun FB, Leemans A, Tax CMW, Biessels GJ, Stieltjes B et al (2014) Methodological considerations on tract-based spatial statistics (TBSS). Neuroimage 100:358-369.

30. Squarcina L, Houenou J, Altamura AC, Soares J, Brambilla P (2017) Association of increased genotypes risk for bipolar disorder with brain white matter integrity investigated with tract-based spatial statistics. J Affect Disorders 221:312-317.

31. Rizk MM, Rubin-Falcone H, Keilp J, Miller JM, Sublette ME, Burke A et al (2017) White matter correlates of impaired attention control in major depressive disorder and healthy volunteers. J Affect Disorders 222:103-111.

32. Michielse $\mathrm{S}$, Gronenschild E, Domen $\mathrm{P}$, van Os J, Marcelis M, Genetic Risk Outcome P (2017) The details of structural disconnectivity in psychotic disorder: a family-based study of non-FA diffusion weighted imaging measures. Brain Res 1671:121-130.

33. Lukoshe A, van den Bosch GE, van der Lugt A, Kushner SA, Hokken-Koelega ACWhite T (2017) Aberrant white matter microstructure in children and adolescents with the subtype of Prader-Willi syndrome at high risk for psychosis. Schizophrenia Bull 43:1090-1099.

34. Drew DA, Koo B-B, Bhadelia R, Weiner DE, Duncan S, Mendoza-De la Garza $M$ et al (2017) White matter damage in maintenance hemodialysis patients: a diffusion tensor imaging study. BMC Nephrol 18(1):213.

35. Chen F, Chen F, Shang Z, Shui Y, Wu G, Liu C et al (2017) White matter microstructure degenerates in patients with postherpetic neuralgia. Neurosci Lett 656:152-157.

36. Xie Y, Zhang Y, Qin W, Lu S, Ni CZhang Q (2017) White matter microstructural abnormalities in type 2 diabetes mellitus: a diffusional kurtosis imaging analysis. AJNR Am J Neuroradiol 38:617-625.

37. Siddiqui MN, Siddiqui S, Ranasinghe JS, Furgang FA (2002) Pain Management: Trigeminal Neuralgia. Physician 39:64-70.

38. Taylor KS, Anastakis DJ, Davis KD (2009) Cutting your nerve changes your brain. Brain J. Neurol 132:3122-3133.

39. Davis KD, Taylor KS, Anastakis DJ (2011) Nerve injury triggers changes in the brain. Neuroscientist 17:407-422 A Review Journal Bringing Neurobiology Neurology \& Psychiatry.

40. Boretius S, Escher A, Dallenga T, Wrzos C, Tammer R, Brück W et al (2012) Assessment of lesion pathology in a new animal model of MS by multiparametric MRI and DTI. Neuroimage 59:2678-2688.

41. Beaulieu C (2002) The basis of anisotropic water diffusion in the nervous system - a technical review. NMR Biomed 15:435-455.

42. Zatorre RJ, Fields RD, Johansenberg H (2012) Plasticity in gray and white: neuroimaging changes in brain structure during learning. Nat Neurosci 15 $528-536$. 
43. Parise M, Almodovar Kubo TT, Doring TM, Tukamoto G, Vincent MGasparetto EL (2014) Cuneus and fusiform cortices thickness is reduced in trigeminal neuralgia. J Headache Pain 15:17.

44. Abarca-Olivas J, Feliu-Rey E, Sempere AP, Sanchez-Paya J, Bano-Ruiz E, Angel Caminero-Canas M et al (2010) Volumetric measurement of the posterior fossa and its components using magnetic resonance imaging in idiopathic trigeminal neuralgia. Rev Neurol 51:520-524.

45. DeSouza DD, Moayedi M, Chen DQ, Davis KD, Hodaie M (2013)

Sensorimotor and pain modulation brain abnormalities in trigeminal neuralgia: a paroxysmal, sensory-triggered neuropathic pain. PLoS One 8(6): e66340.

46. Obermann M, Rodriguez-Raecke R, Naegel S, Holle D, Mueller D, Yoon M-S et al (2013) Gray matter volume reduction reflects chronic pain in trigeminal neuralgia. Neuroimage 74:352-358.

47. Obermann M, Midler D, Zwarg T, Gizewski ER, Rodriguez-Racke R, Diener HC et al (2009) Gray matter decrease in patients with trigeminal neuralgia. Neurology 72:A251-AA51.

48. Moisset X, Villain N, Ducreux D, Serrie A, Cunin G, Valade D et al (2011) Functional brain imaging of trigeminal neuralgia. Eur J Pain 15:124-131.

49. Malfliet A, Coppieters I, Van Wilgen P, Kregel J, De Pauw R, Dolphens M et al (2017) Brain changes associated with cognitive and emotional factors in chronic pain: a systematic review. Eur J Pain 21:769-786.

50. Hayes DJ, Chen DQ, Zhong JD, Lin A, Behan B, Walker M et al (2017) Affective circuitry alterations in patients with trigeminal neuralgia. Front Neuroanat 11:73

\section{Submit your manuscript to a SpringerOpen ${ }^{\circ}$ journal and benefit from:}

- Convenient online submission

- Rigorous peer review

- Open access: articles freely available online

- High visibility within the field

- Retaining the copyright to your article

Submit your next manuscript at $>$ springeropen.com 